\title{
GREEDY ALGORITHMS IN COMBINATORIAL OPTIMIZATION
}

\author{
Ulrich Faigle, Twente University of Technology, Enschede \\ The Netherlands
}

The greedy algorithm is perhaps the intuitively most natural optimization principle: take in each step the locally best decision, where "best" is measured by an objective function that is evaluated locally. The question, then, arises under what conditions such a local strategy leads to a globally optimal solution. Particular attention has therefore been payed to combinatorial structures for which the greedy algorithm works provably optimally (at least relative to certain types of objective functions): for example, matroids, polymatroids and their generalizations (e.g., greedoids).

The greedy approach is often quite successfully employed not as a full algorithm but as a fast subroutine within other algorithmic frameworks (e.g., pruning rule in branch-and-bound procedures or local optimizer in a Lagrange relaxation). In general, the greedy algorithm may be seen as a direct attempt to find local optima in search procedures. The greedy strategy appears to be of particular importance in the design of on-line algorithms (e.g., coloring, partitioning, scheduling), where it is often known as the first fit principle.

Recent interest in probabilistic aspects of optimization has exhibited the greedy strategy to be quite powerful in the design of deterministic algorithms based on the evaluation of conditional probabilities (derandomization technicues) or in the analysis of average case behavior (TSF, graph coloring).

This survey lecture cannot give a full treatment of all aspects of greedy principles in combinatorial optimization but tries to sketch some of the results and recent developments in the aforementioned areas. 